\title{
NON-ORTHOGONALISABLE VECTOR FIELDS ON SPHERES
}

\author{
by MARTIN RAUSSEN
}

(Received 26th January 1984)

A $(k-1)$-field on $S^{n-1}$ may be given as a section $\varphi$ of the fibre bundle

$$
p_{n, k}: V_{n, k} \downarrow S^{n-1}
$$

with fibre $V_{n-1, k-1}$, or, equivalently, as a semi-orthogonal map, i.e., a map

$$
\varphi^{\prime}: \mathbb{R}^{n} \times \mathbb{R}^{k} \rightarrow \mathbb{R}^{n},
$$

which is isometric in the second variable and such that for the basis vector $e_{1} \in \mathbb{R}^{k}$ and every $x \in \mathbb{R}^{n}$

$$
\varphi^{\prime}\left(x, e_{1}\right)=x
$$

A section $\varphi$ of $p_{n, k}$ induces a semi-orthogonal map by adjunction, i.e.,

$$
\varphi^{\prime}(x, v)= \begin{cases}0 & x=0 \\ \varphi\left(\|x\|^{-1} \cdot x\right)(\|x\| \cdot v) & x \neq 0\end{cases}
$$

A semi-orthogonal map $\mu$ determines a section

$$
\operatorname{ad}(\mu): S^{n-1} \longrightarrow V_{n, k}
$$

of $p_{n, k}$ by adjunction and restriction [12].

A $(k-1)$-field $\varphi$ on $S^{n-1}$ is called skew iff

$$
\varphi^{\prime}(-x, v)=-\varphi^{\prime}(x, v) .
$$

$\varphi$ is called orthogonal iff $\varphi^{\prime}$ is an orthogonal multiplication, i.e., iff $\varphi^{\prime}$ is isometric in both variables. Obviously, orthogonal $r$-fields are skew. An $r$-field $\varphi$ is called orthogonalisable iff it is regularly homotopic to an orthogonal one, and non-orthogonalisable otherwise.

Skew $r$-fields on spheres were studied by Milgram and Zvengrowski. They show that every $r$-field on a sphere is regularly homotopic to a skew $r$-field [9]. They also remark that, in general, not every vector field on a sphere is orthogonalisable. We give some precise conditions for the existence of such non-orthogonalisable vector fields. 
Some more notational conventions: For a fibre bundle

$$
p: E \downarrow B
$$

the space of sections of $p$ will be denoted by $\Gamma(p)$. Let

$$
o_{n-1}, l_{n-1} \in \pi_{n-1}\left(S^{n-1}\right)
$$

denote the homotopy class of the trivial map, resp. the indentity, and

$$
\left(p_{n, k^{*}}\right)^{-1}\left(o_{n-1}\right), \text { resp. }\left(p_{n, k^{*}}\right)^{-1}\left(l_{n-1}\right) \subseteq \pi_{n-1}\left(V_{n, k}\right)
$$

their preimages in the homotopy exact sequence associated to $p_{n, k}$. Notice that inclusion induces the maps

$$
\begin{gathered}
\sigma_{0}: \pi_{n-1}\left(V_{n-1, k-1}\right) \stackrel{\sim}{\rightarrow}\left(p_{n, k^{*}}\right)^{-1}\left(o_{n-1}\right) \subseteq \pi_{n-1}\left(V_{n, k}\right) \\
\sigma_{1}: \pi_{0}\left(\Gamma\left(p_{n, k}\right)\right) \rightarrow\left(p_{n, k^{*}}\right)^{-1}\left(l_{n-1}\right) \subseteq \pi_{n-1}\left(V_{n, k}\right) .
\end{gathered}
$$

Lemma 1. If $\Gamma\left(p_{n, k}\right)$ is non-empty, $\sigma_{1}$ is a bijection. As a consequence, there is a bijection

$$
\alpha: \pi_{n-1}\left(V_{n-1, k-1}\right) \rightarrow \pi_{0}\left(\Gamma\left(p_{n, k}\right)\right)
$$

such that

$$
\sigma_{1}(\alpha(x))=\sigma_{0}(x)+\sigma_{1}(\alpha(0))
$$

Proof. According to [7], Lemma 1.1, the map $\sigma_{0}$ is injective. As $p_{n, k}$ is a fibration, every homotopy into the base space $S^{n-1}$ ending with the identity map can be lifted to a homotopy into the total space ending with a section.

According to Eckmann [4], every orthogonal multiplication

$$
\mu: \mathbb{R}^{k} \times \mathbb{R}^{n} \rightarrow \mathbb{R}^{n}
$$

arises from a representation of the Clifford group $C_{k-1}$. If $k \neq 0(4)$, there is a welldetermined $a_{k}$-dimensional irreducible $C_{k-1}$-representation $M_{k}$; if $k \equiv 0(4)$, there are exactly two non-equivalent irreducible representations $M_{k}^{0}, M_{k}^{1}$. Here $a_{k}$ is given by

$$
a_{1}=1, a_{2}=2, a_{3}=a_{4}=4, a_{5}=a_{6}=a_{7}=a_{8}=8 \text {, and } a_{k+8}=16 a_{k} \text {. }
$$

Thus, for $n, k$ as above, $n=i \cdot a_{k}, i>0$, every $n$-dimensional $C_{k-1}$-representation is equivalent to

(a) $i \cdot M_{k}, k \neq 0(4)$

(b) $a \cdot M_{k}^{0} \oplus b \cdot M_{k}^{1}, a+b=i, k \equiv 0(4)$. 
The corresponding orthogonal multiplications, which are well-determined up to $0(n)$ conjugation, will be denoted by $\mu(i)$, resp. $\mu(a, b)$. We are interested in the adjoints of representatives $\mu(i), \mu(a, b)$ :

$$
\operatorname{ad}(\mu(i)), \operatorname{ad}(\mu(a, b)): S^{n-1} \rightarrow V_{n, k} .
$$

Obviously, conjugation by a rotation does not alter the homotopy class of such a vector field, whereas a non-rotation might do so [6]. A simple counting argument shows:

Proposition 1. If $a_{k}$ divides $n$, and

(a) $k \neq 0(4)$ and $\left|\pi_{n-1}\left(V_{n-1, k-1}\right)\right|>2$ or

(b) $k \equiv 0$ (4) and $\left|\pi_{n-1}\left(V_{n-1, k-1}\right)\right|>2\left(\left(n / a_{k}\right)+1\right)$,

then there is a non-orthogonalisable $(k-1)$-field on $S^{n-1}$.

In general, it is difficult to determine these homotopy groups. Therefore, we study the boundary homomorphism

$$
\partial: \pi_{m-1}\left(V_{s, r}\right) \rightarrow \pi_{m-2}\left(S^{s-r-1}\right)
$$

corresponding to the fibre bundle

$$
S^{s-r-1} \rightarrow V_{s, r+1} \downarrow V_{s, r}
$$

in the cases $(s, r)=(n-1, k-1)$, resp. $(n, k)$.

$\partial$ will be applied to the adjoints

$$
\operatorname{ad}(\mu): S^{n-1} \rightarrow V_{n, k}
$$

of semi-orthogonal maps

$$
\mu: \mathbb{R}^{k} \times \mathbb{R}^{n} \rightarrow \mathbb{R}^{n}
$$

Note that for an orthogonal map $\mu$, there is also an adjunction

$$
\operatorname{ad}^{\prime}(\mu): S^{k-1} \rightarrow 0(n)
$$

We also make use of the generalised $J$-homomorphisms [12]

$$
J_{r}^{s}: \pi_{m}\left(V_{s, r}\right) \rightarrow \pi_{m+r}\left(S^{s}\right)
$$

The following propositions are the tools in our argument:

Proposition 2. [12] If $n \equiv k(2)$ and $n>2 k+1$, then

$$
2 \cdot \pi_{k-1}^{s} \subseteq \sum^{\infty} \partial\left(\pi_{n-1}\left(V_{n-1, k-1}\right)\right) \subset \pi_{k-1}^{s} .
$$


Proposition 3. [6][11]. For $r<s$, the diagram

commutes.

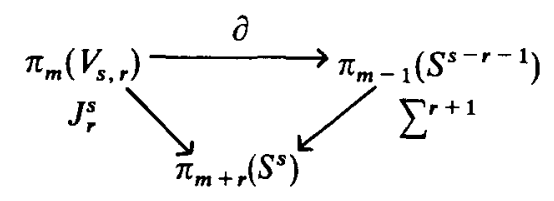

Lemma 2. For an orthogonal map

$$
\mu: \mathbb{R}^{n} \times \mathbb{R}^{k} \rightarrow \mathbb{R}^{n},
$$

the following relation holds:

$$
J_{k}^{n}(\operatorname{ad}(\mu))=(-1)^{k n} J\left(\operatorname{ad}^{\prime}(\mu)\right) \in \pi_{n+k-1}\left(S^{n}\right) .
$$

Our main result is:

Proposition 4. If $a_{k}$ divides $n, 2 k+1<n$, and

(a) $k \equiv 2$ (4) and 2-coker $J_{k-1} \neq 0$ or

(b) $k \equiv 0$ (4) and $2 \cdot \operatorname{coker} J_{k-1} \neq 0$ or

$$
2 \cdot\left(\frac{n}{a_{k}}+1\right)<\left|\operatorname{im} J_{k-1}\right|
$$

then there exists a non-orthogonalisable $(k-1)$-field on $S^{n-1}$.

Proof. Obviously, the diagram

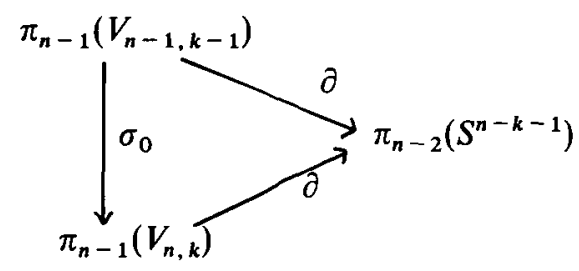

commutes. Using Lemma 1 and Proposition 2, it follows that for any $\varphi_{0} \in \pi_{0}\left(\Gamma\left(p_{n, k}\right)\right)$, serving as a base point,

$$
\sum^{\infty} \partial\left(\sigma_{1}\left(\pi_{0}\left(\Gamma\left(p_{n, k}\right)\right)\right) \supseteq \sum^{\infty} \partial \sigma_{1}\left(\varphi_{0}\right)+2 \cdot \pi_{k-1}^{s} .\right.
$$

According to Lemma 2, we get for every orthogonal map

$$
\mu: \mathbb{R}^{n} \times \mathbb{R}^{k} \rightarrow \mathbb{R}^{n}
$$


that

$$
\partial(\operatorname{ad}(\mu))=J_{k}^{n}(\operatorname{ad}(\mu))=(-1)^{k n} J\left(\operatorname{ad}^{\prime}(\mu)\right)
$$

thus

$$
\sum^{\infty} \partial(\operatorname{ad}(\mu)) \in \operatorname{im}\left(J_{k-1}\right)
$$

Thus, the proposition is already proved if $2 \cdot$ coker $J_{k-1} \neq \emptyset$. In the last case, we use the calculation of $K 0$-groups via Clifford forms in [2]. The authors show that, for $k<n$,

$$
\operatorname{ad}^{\prime}(\mu(a, b))=(a-b) \cdot g \in \pi_{k-1}(0(n)) \cong \pi_{k-1}(0)
$$

where $g \in \pi_{k-1}(0) \cong \mathbb{Z}$ is a generating element. According to Proposition 2 ,

$$
\sum^{\infty} \partial\left(\sigma_{1}\left(\pi_{0}\left(\Gamma\left(p_{n, k}\right)\right)\right) \supseteq \frac{n}{a_{k}} \cdot J(g)+2 \cdot \operatorname{im} J_{k-1} \cdot\right.
$$

On the other hand

$$
\begin{aligned}
\left\{\sum^{\infty} \partial(\operatorname{ad}(\mu(a, b)) \mid a+b\right. & \left.=\frac{n}{a_{k}}\right\}=\left\{J\left(\operatorname{ad}^{\prime}(\mu(a, b))\right) \mid a+b=\frac{n}{a_{k}}\right\} \\
& =\left\{i \cdot J(g) \mid-\frac{n}{a_{k}} \leqq i \leqq \frac{n}{a_{k}} ; i \equiv \frac{n}{a_{k}} \bmod 2\right\} .
\end{aligned}
$$

Thus, under the second condition in Proposition 4(b), $\partial$ applied to orthogonal forms cannot fill out the image of $\partial$ applied to semi-orthogonal forms.

Corollary. (a) For $m \leqq 46$, there is a non-orthogonalisable 3-field on $S^{4 m-1}$.

(b) For $q$ odd, there exist non-orthogonalisable 23-fields on $S^{9^{-2}{ }^{11}-1}$.

Proof. (a) $\pi_{3}\left(V_{3,3}\right)=\pi_{3}(0(3))=\mathbb{Z}$; from Paechter's tables [10], we find that, $\pi_{4 l-1}\left(V_{4 l-1,3}\right)=\mathbb{Z} / 24 \oplus \mathbb{Z} / 4, l \geqq 2$. Apply Proposition 1 .

(b) An inspection of Toda's tables [13], [14] and [1] shows that coker $J_{23} \cong \mathbb{Z} / 3$. Apply Proposition 4.

The same methods are useful to compare spaces of skew-linear, non-singular bilinear and orthogonal maps (see [8], [3]). Two skew-linear maps $\varphi_{0}, \varphi_{1}$ are called homotopic, if there is a continuous path in the space of all skew-linear maps from $\varphi_{0}$ to $\varphi_{1}$; etc.

Proposition 5. (a) For $k, n$ as in Proposition 1 or 4 there exists a skew-linear form

$$
\varphi: \mathbb{R}^{n} \times \mathbb{R}^{k} \rightarrow \mathbb{R}^{n},
$$

which is not homotopic to an orthogonal form. 
(b) If $2 \cdot \operatorname{coker} J_{k-1} \neq 0$ and $a_{k}$ divides $n$, there exists $N \geqq 0$ and a non-singular bilinear form

$$
\varphi: \mathbb{R}^{n} \times \mathbb{R}^{k+N} \rightarrow \mathbb{R}^{n+N}
$$

which is not homotopic to an orthogonal form.

Remark. Thus, in these cases, even the sets of components of the spaces of skewlinear, bilinear and orthogonal forms are different.

Proof. For a semi-orthogonal map

$$
\varphi: \mathbb{R}^{k} \times \mathbb{R}^{n} \rightarrow \mathbb{R}^{n},
$$

the Hopf-construction is given by

$$
H(\varphi)=J_{k}^{n}(\operatorname{ad}(\varphi))=\sum^{\infty} \partial(\operatorname{ad}(\varphi)) \in \pi_{k-1}^{s} .
$$

Thus, the Hopf-construction applied to all semi-orthogonal forms yields just the set $\sum^{\infty} \partial\left(\pi_{n-1}\left(V_{n, k}\right)\right)$, whereas applying it to only orthogonal forms gives us just the subset described in the proof of Proposition 4.

(a) is now proved by representing every regular homotopy class of $(k-1)$-fields on $S^{n-1}$ by a skew $(k-1)$-field $\varphi$ [9] and regarding the skew-linear form $\varphi^{\prime}$. In (b) we use Lam's trick [8] to find a non-singular bilinear form

$$
\varphi_{N}^{\prime}: \mathbb{R}^{n} \times \mathbb{R}^{k+N} \rightarrow \mathbb{R}^{n+N}
$$

by Clifford suspensions applied to $\varphi$. This process does not alter the Hopf-construction. On the other hand, a result of $\mathrm{H}$. Hefter [5] shows, that

$$
H(\psi) \in \operatorname{im} J_{a+b-c-1}
$$

for every orthogonal form

$$
\psi: \mathbb{R}^{a} \times \mathbb{R}^{b} \rightarrow \mathbb{R}^{c} .
$$

I would like to thank the Instituto de Matematicas of the UNAM, Mexico City for their kind hospitality during my stay, when a first draft of this paper was written up.

Furthermore, I gratefully acknowledge helpful remarks by M. Audin (Paris), Th. Bier (Göttingen) and E. Ossa (Wuppertal) and the referee.

\section{REFERENCES}

1. J. F. Adams, On the groups $J(X)$ IV, Topology 5 (1966), 21-71.

2. M. F. Atryah, R. Bott and A. Shapiro, Clifford modules, Topology 3 (Supplement 1) (1964), 3-38. 
3. Th. Bier and U. Schwardmann, Räume normierter Bilinearformen und Cliffordstrukturen, Math. Z. 180 (1982), 203-215.

4. B. Eckmann, Gruppentheoretischer Beweis des Satzes von Hurwitz-Radon über die Komposition quadratischer Formen, Comment. Math. Helv. 15 (1943), 358-366.

5. H. Hefter, Dehnungsuntersuchungen an Sphärenabbildungen, Invent. Math. 66 (1982), 1-10.

6. I. M. JAMEs, The topology of Stiefel manifolds (Cambridge University Press, 1976).

7. I. M. James and E. Thomas, Note on the classification of cross-sections, Topology 4 (1966), $351-359$.

8. K. Y. LAM, On the bilinear and skew-linear maps that are non-singular, Quart. J. Oxford 19 (1968), 281-288. 336.

9. R. J. Milgram and P. Zvengrowski, Skewness of $r$-fields on spheres, Topology 15 (1976), 325-

10. G. F. PAEChter, The groups $V_{n, m}-I$, Quart. J. of-Math. Oxford 7 (1956), 249-269.

11. M. Raussen and L. SMith, A geometric interpretation of sphere bundle boundaries and generalized $J$-homomorphisms with an application to a diagram of I. M. James, Quart. J. Oxford 30 (1979), 113-117.

12. L. Sмiтh, Nonsingular bilinear forms, generalized $J$-homomorphisms and the homotopy of spheres I, Ind. U. Math. J. 27 (1978), 697-737.

13. H. Toda, p-primary components of homotopy groups III. Stable groups of the sphere, Mem. Coll. Sci. Univ. Kyoto Ser. A Math. 31 (1958), 191-210.

14. H. TodA, Composition method in homotopy groups of spheres (Ann. of Math.) Studies 49, Princeton University Press, 1962.

Mathematissches Institut der Universität Góttingen

Bunsenstrasse 3/5, D 3400 GötTingen

AND

Matematisk Institut, Aarhus Universitet

Ny Munkegade, DK-8000 Aarhus C

Current Address:

INSTITUT FOR ELEKTRONISKe SySTEMER

AALBORG UNIVERSITETSCENTER

Strandvejen 19, DK 9000 Aalborg 